\title{
Preparación de secciones estratigráficas: aspectos prácticos del análisis de estratos en obras del Patrimonio Cultural (pigmentos y soportes)
}

\author{
E. JIMENEZ ROCA, A. RUIZ-CONDE, P.J. SANCHEZ-SOTO
}

Instituto de Ciencia de Materiales de Sevilla (ICMSE), Centro Mixto CSIC-US, c/Américo Vespucio n 49 , Parque Científico-Tecnológico Cartuja'93, Isla de la Cartuja, 41092-Sevilla

\begin{abstract}
Las secciones estratigráficas (estratigrafías) son de suma utilidad para los estudios científicos de muestras pertenecientes al Patrimonio Cultural. En este artículo se pretende dar una serie de consejos y orientaciones útiles como metodología de trabajo que permite un fácil, económico y versátil procedimiento de preparación de dichas secciones estratigráficas, con posibilidad de realización simultánea. En concreto, las distintas etapas a seguir son: a) preparación inicial utilizando tiras de (poli)metacrilato; b) embutido de las muestras; c) corte de las preparaciones; d) lijado y pulido; e) observación al microscopio óptico y fotografiado.

Se describen los aspectos prácticos más destacados en el análisis de estratos que se pueden realizar; en particular, los pigmentos y soportes como ejemplos más ilustrativos. Se destaca la importancia de la obtención de estratigrafías representativas si lo que se pretende es llegar a un diagnóstico o aproximación al estado de alteración-degradación de la obra de arte con objeto de una fase siguiente de restauración y conservación de la misma, a cargo de otros especialistas. Asimismo, la preparación de estratigrafías abre la posibilidad de estudios posteriores por distintas técnicas instrumentales de análisis de materiales, obteniendo datos de relevancia sobre textura, composición química y de fases de muestras del Patrimonio Cultural.
\end{abstract}

Palabras clave: sección estratigráfica, Patrimonio Cultural, análisis de estratos, pigmentos, soportes.

\begin{abstract}
Preparation of stratigraphic sections (cross-sections): some practical aspects on the analysis of strates coming from Cultural Heritage materials (pigments and supports)
\end{abstract}

The stratigraphic sections (cross-sections) are of valuable utility for scientific studies concerning samples coming from Cultural Heritage materials. This paper aims to show several indications and orientations as a working methodology that allows an easy, economic and versatile procedure of simultaneous preparation concerning these stratigraphic sections. Several steps must be followed: a) initial preparation of samples using (poly)metacrylate bars; b) consolidation using a polymer; c) cutting; d) polishing; e) observation using optical microscopy and photography.

The main practical aspects involved in the stratigraphic analysis are described. In particular, pigments and supports are used as illustrative examples. It is pointed out the importante of stratigraphic preparations, which must be representative of the material if a diagnosis or approximation to the conservation-degradation state of the original artwork piece is being pursued. This is very important prior to a second step of restauration and conservation by other specialists. Moreover, the preparation of stratigraphic sections open the possibility of further studies using several techniques applied in materials science analysis, obtaining relevant results concerning texture, chemical and phase composition of these samples of Cultural Heritage.

Keywords: stratigraphic section (cross-section), Cultural Heritage, analysis of strates, pigments, supports

\section{INTRODUCCIÓN}

El Patrimonio Histórico y Cultural es un área de conocimiento de gran crecimiento en todos los órdenes por su creciente importancia y las implicaciones que posee, de forma directa, en la propia sociedad. A juicio de los expertos (1), el concepto de Patrimonio es, por necesidad, demasiado amplio, con denominaciones que vuelven cíclicamente como un ejercicio de sinonimia (Tesoro Cultural, Patrimonio HistóricoArtístico, Bienes Culturales, etc.) y es un referente cada vez más necesario a la política de intereses de cada pueblo o grupo para reivindicar sus imágenes culturales.

En el CSIC se ha constituido una Red Temática de Patrimonio Histórico y Cultural (RTPHC del CSIC) en octubre de 2001. En dicha Red Temática se adscriben diversos grupos de investigación en áreas como son Materiales, Física, Geología, Arqueología, etc. que se ocupan de la realización de este tipo de estudios con el objetivo, además, de llevarlos a cabo entre los diversos grupos de especialistas de la Red. Se trataría de estimular una cooperación multidisciplinar entre especialistas de las Ciencias Humanísticas y de las Ciencias Experimentales. Durante los últimos años se hace cada vez más patente que es necesaria la colaboración de los propios historiadores y arqueólogos con los físicos, geólogos y químicos, pero además es imprescindible contar con especialistas en una gran variedad de materiales y disponer de una serie de técnicas específicas de investigación y estudio (2-4).

Los primeros investigadores que se ocuparon del estudio de las obras de arte ya observaron las ventajas que suponía el análisis de los estratos presentes y empezaron a poner a punto técnicas para la obtención de una sección transversal 
de muestras suministradas por los especialistas, como son los de Bellas Artes, Historia y Arqueología (5-7). Con el análisis estratigráfico de trozos muy pequeños de muestras representativas de obras de arte, se puede conseguir una amplia visión directa que facilita el estudio e investigación de los diferentes estratos o capas que se pueden encontrar en un material dado, puesto que la mayoría de obras posee una estructura de estratos superpuestos que la conforman. El caso más claro es el de una obra pictórica. La cantidad de información que se puede obtener es muy valiosa, por ejemplo datos sobre secuencia de las capas, técnicas de ejecución y estado de conservación y puede compensar, en parte, el posible daño que se realiza con la obtención de la muestra directamente de la obra (3-7). Los estratos más externos son visibles, pero pueden ser tan importantes o más los estratos supeditados que le sirven de soporte y de base: el análisis estratigráfico es capaz de ponerlos de manifiesto. A veces, los propios autores del estudio estratigráfico han sido los que han tomado también las muestras directamente in situ bajo consejo y dirección de otros especialistas $(9,10)$. En general, se prefiere una muestra pequeña, pero completa, que una más grande o parcial o bien que manifieste tendencia a fragmentarse paralelamente a los estratos.

El contenido eminentemente práctico de este artículo servirá para hacerse una idea amplia de lo que supone preparar la estratigrafía de una muestra desde su recepción hasta llegar a observarla con lupa o microscopio óptico y fotografiarla, así como disponer de la misma para estudios posteriores mediante la aplicación de otras técnicas instrumentales de análisis de materiales. Se expone la metodología para la obtención de estratigrafías que se viene siguiendo, desde hace años, en el seno del grupo de investigación al que pertenecen los autores. Asimismo, se dan una serie de consejos y orientaciones para proceder, con especial cuidado, a lo largo de todo el proceso de obtención de la sección en una muestra, particularizando al caso ilustrativo de pigmentos y soportes.

Existen diferentes formas de preparación de muestras estratigráficas (5-8). Para la obtención de una sección estratigráfica de una muestra muy pequeña y heterogénea, con una consistencia generalmente frágil, es necesario realizar una inclusión de dicha muestra empleando una resina o polímero que sirva de soporte para proceder a ciertas manipulaciones mecánicas. Sin embargo, con el sistema descrito a continuación se consiguen una serie de ventajas, como es sencillez, economía de medios, fiabilidad, rapidez, reproducibilidad, realización de varias preparaciones simultáneas, etc. No obstante, las diferentes etapas que conlleva hay que realizarlas con sumo cuidado, puesto que un fallo en cualquiera de ellas puede suponer el deterioro parcial o total de la muestra del Patrimonio Cultural, en general muy valiosa y disponible en muy pequeña cantidad, con el consiguiente perjuicio que puede ocasionar.

\section{METODO EXPERIMENTAL}

\subsection{Preparación inicial}

En primer lugar han de realizarse una serie de taladros en forma de cono, empleando una broca de $8 \mathrm{~mm}$, utilizando unas tiras de metacrilato transparente (polimetacrilato de metilo) de unos $10 \mathrm{~mm}$ de ancho, cortadas de una plancha (8-10 $\mathrm{mm}$ de espesor) y que servirán para contener las muestras. Dichos taladros deben estar lo más próximo posible, pero sin llegara a afectarse entre ellos y sin perforar completamente el metacrilato, limpiando los restos de polvo o viruta acumulados.

\subsection{Embutido de las muestras}

Se recomienda usar un compuesto acrílico e incoloro, de fraguado rápido, de los utilizados comúnmente por los protésicos dentales. Uno de ellos es el Palopress ${ }^{\circledR}$ Vario, Clear 7, en polvo, de la casa Heraus-Kulzer (Laboratory Products Division) y la resina líquida Cold-curing Palopress ${ }^{\circledR}$ for precision cast partial dentures, que contiene metacrilato de metilo y que se denominará en adelante como "catalizador".

Delante de las muestras alineadas, se coloca la tira perforada de metacrilato sobre un papel de filtro. Comenzando por uno de los extremos de la misma, se introduce una pequeña cantidad de resina en polvo hasta cubrir, aproximadamente, la mitad del cono dejado por la broca en los orificios y de los que previamente se han eliminado los restos de polvo y virutas. Mediante una pipeta o cuentagotas, se deja caer una gota de catalizador en cada uno estos conos y se espera de 30 a 60 segundos, dependiendo de la temperatura ambiente. Con una pinza, se introduce la muestra a estudiar, a ser posible con la capa externa hacia abajo. A continuación, se añade catalizador con la pipeta y polvo de resina en dos o tres pasos hasta conseguir, aproximadamente, el máximo nivel de altura dado por el espesor de la tira de metacrilato (Figura 1). En caso que las muestras sean algo porosas o de tamaño considerable, proporcional al cono, como fase previa es conveniente impregnarlas aparte utilizando una placa-gotero de porcelana vidriada con el mismo catalizador, al objeto de evitar la formación de burbujas. Es muy aconsejable esperar unas horas o mejor toda la noche, hasta el día siguiente, antes de iniciar el paso siguiente.

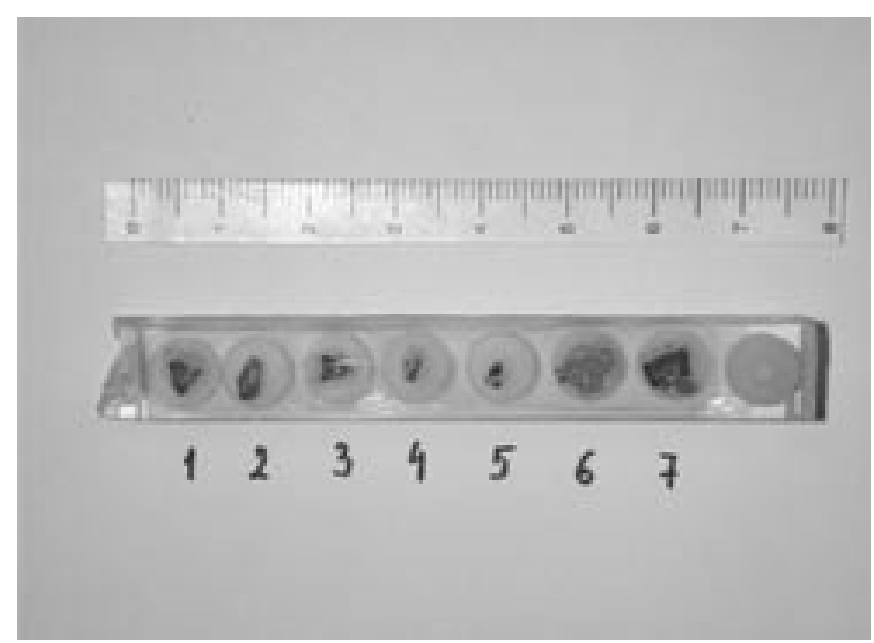

Fig.1.- Fotografía de una serie de muestras de pigmentos y soportes procedentes de diversas pinturas murales, realizadas simultáneamente, ya embutidas con resina en la barra de metacrilato, dispuestas para la preparación de estratigrafías individuales.

\subsection{Corte de las preparaciones}

Se sujeta la tira de metacrilato con las muestras embutidas en un tornillo-mordaza y se inicia el corte con una sierra. En todo caso, ha de hacerse este corte perpendicular y próximo a la muestra para conseguir observar el espesor real de los 
estratos y lijar lo menos posible, respectivamente. El corte ha de realizarse con cuidado, firmeza y despacio, para evitar que el rozamiento y el calor que se produce ocasionen posibles daños.

\subsection{Lijado y pulido}

El lijado se puede hacer de forma manual sobre una mesa o plataforma limpia sin rugosidades y sin partículas que estorben, o bien con una lijadora orbital. Con papel de lija de grano 400 (SiC Buehler), se pasará la primera muestra y sobre la cara que interese, hasta conseguir que aparezca levemente la misma. La cara contraria se lijará también, procurando corregir el paralelismo de ambas para evitar desenfoques innecesarios en el microscopio. El siguiente paso sería la misma operación anterior, pero con papel de grano 1200. Por último, se pule la muestra embutida con un paño o fieltro de hilo. Por otra parte, si persistieran algunos arañazos después del pulido en las preparaciones, se puede dar en la superficie de éstas un poco de Bálsamo del Canadá (utilizado en microscopía óptica convencional) empleando un pincel.

\subsection{Observación al microscopio óptico o lupa y fotografiado}

Los autores han examinado distintas preparaciones estratigráficas empleando un microscopio óptico Olimpus con iluminación blanca de luz reflejada y cámara fotográfica convencional y mediante fotografía digital empleando una cámara Nikon Coolpix, adaptándola a dicho microscopio

Las observaciones al microscopio permiten apreciar la propia estructura estratigráfica, los colores de las distintas capas donde aparecen pigmentos y soportes, su forma, aspectos texturales, espesor de las capas, inclusiones presentes de distinto tamaño de partículas, etc. En el apartado siguiente se presentan algunos ejemplos de preparaciones estratigráficas donde pueden apreciarse varios estratos y soportes.

\section{RESULTADOS OBTENIDOS: ALGUNOS EJEMPLOS ILUSTRATIVOS}

Al disponer de preparaciones estratigráficas en secciones transversales de las distintas muestras, éstas se pueden examinar por técnicas instrumentales de análisis de materiales.
Ya se ha mencionado la microscopía óptica, pero también se pueden estudiar directamente mediante espectroscopía de infrarrojos (IR), sobre todo si está provisto el equipo con un microscopio de infrarrojos. En el modo de reflexión, se precisa una superficie lisa, de manera que se puede utilizar la propia muestra si posee una superficie definida. Como las muestras suelen ser irregulares o muy pequeñas, se recurre a embutirla en una resina y a realizar la correspondiente sección estratigráfica pulida para estudiarla por IR, con un grosor mínimo de unos 10 micrómetros, evitando el uso de pastillas de $\mathrm{KBr}$ y muestras sometidas a molienda.

Asimismo, las secciones estratigráficas se pueden estudiar directamente por microscopía electrónica de barrido (MEB) y análisis químico por energías dispersivas de rayos $X$ (EDX acoplado al equipo de MEB) para analizar las distintas capas por separado. Para el estudio de muestras con estas dos últimas técnicas, se recubren las estratigrafías con una fina capa de un metal (metalizado con oro) para hacerlas conductoras y facilitar el examen por MEB. En consecuencia, la preparación de estratigrafías posibilita un examen por distintas técnicas instrumentales de análisis de materiales, con la ventaja de que los resultados que se obtengan estarán, en este caso, asociados a la localización de los materiales en el contexto estratigráfico de los distintos fragmentos (5-12).

A título de ejemplo, en la Figura 2 se presenta una sección estratigráfica a bajo y mayor aumento, donde se pueden apreciar distintas capas de pigmentos y soportes y que corresponde a una pintura mural del Siglo XV con otra más moderna superpuesta, cuyo estudio se está completando en la actualidad y que parece ser se ha realizado con la técnica del fresco (9). Un análisis más detallado se presenta en la microfotografía de la Figura 3a, donde se muestra una estratigrafía de otro fragmento de pintura mural en el cual aparecen distintas capas. Un esquema de su probable composición se da en la Figura 3b, una vez deducido mediante estudios por microscopía electrónica de barrido y análisis químico utilizando EDX, además de espectroscopía IR para identificación de posibles compuestos orgánicos presentes (10). En este caso particular, la estratigrafía que se muestra, de grosor total de unos $1.3 \mathrm{~mm}$, ha permitido la identificación posterior con dichas técnicas instrumentales de una preparación-base de $1 \mathrm{~mm}$ de yeso $\left(\mathrm{CaSO}_{4} \cdot 2 \mathrm{H}_{2} \mathrm{O}\right)$ con cola animal, una capa de pigmentos de unos $0.2 \mathrm{~mm}$ de albayalde (carbonato básico de plomo), calcita y compuestos de cobre (malaquita, carbonato básico de cobre) mezclados con aceite
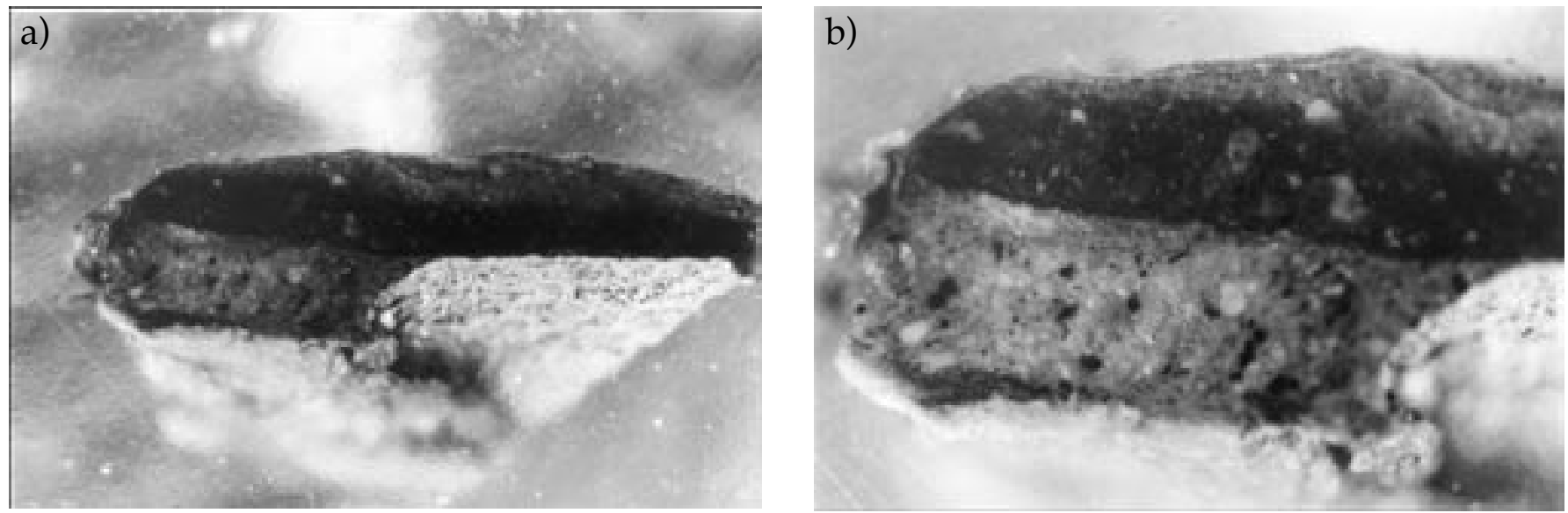

Fig.2.- Microfotografías al microscopio óptico de una preparación estratigráfica (estratigrafía), sección transversal, de un fragmento extraído de una pintura mural, correspondiente al soporte y capa pictórica: a) aumento (x20) y b) aumento (x60). 
de linaza, un posible repinte con una nueva base de $0.2 \mathrm{~mm}$ de yeso con cola; hay otra capa de pigmentos a base de un bol ocre (óxidos de hierro hidratado con cuarzo y arcillas) y aceite de linaza; por último, existe una capa superficial de unos $0.01 \mathrm{~mm}$ de un barniz. Es decir, se pueden poner en evidencia pinturas superpuestas a otras y en este caso, además de los pigmentos y soportes, la técnica de ejecución (pintura al óleo). A veces se encontrado incluso la presencia de delgadas láminas metálicas, por ejemplo de oro, en muestras de esculturas policromadas realizadas en cerámica cocida ("terracotta") que han sido objeto de estudios previos $(8,12)$, además de capas de suciedad y sales producidas por efecto del tiempo y la humedad $(6,9,10,12)$.

De acuerdo con lo expuesto, la preparación de secciones estratigráficas se puede extender también al estudio de materiales cerámicos y vidriados con suma facilidad siguiendo las indicaciones dadas en este artículo, tal y como se ha mencionado para el caso de estas esculturas en cerámica cocida (terracotas), poniendo en evidencia vidriados y pinturas superpuestas, lo que permite estudios posteriores.
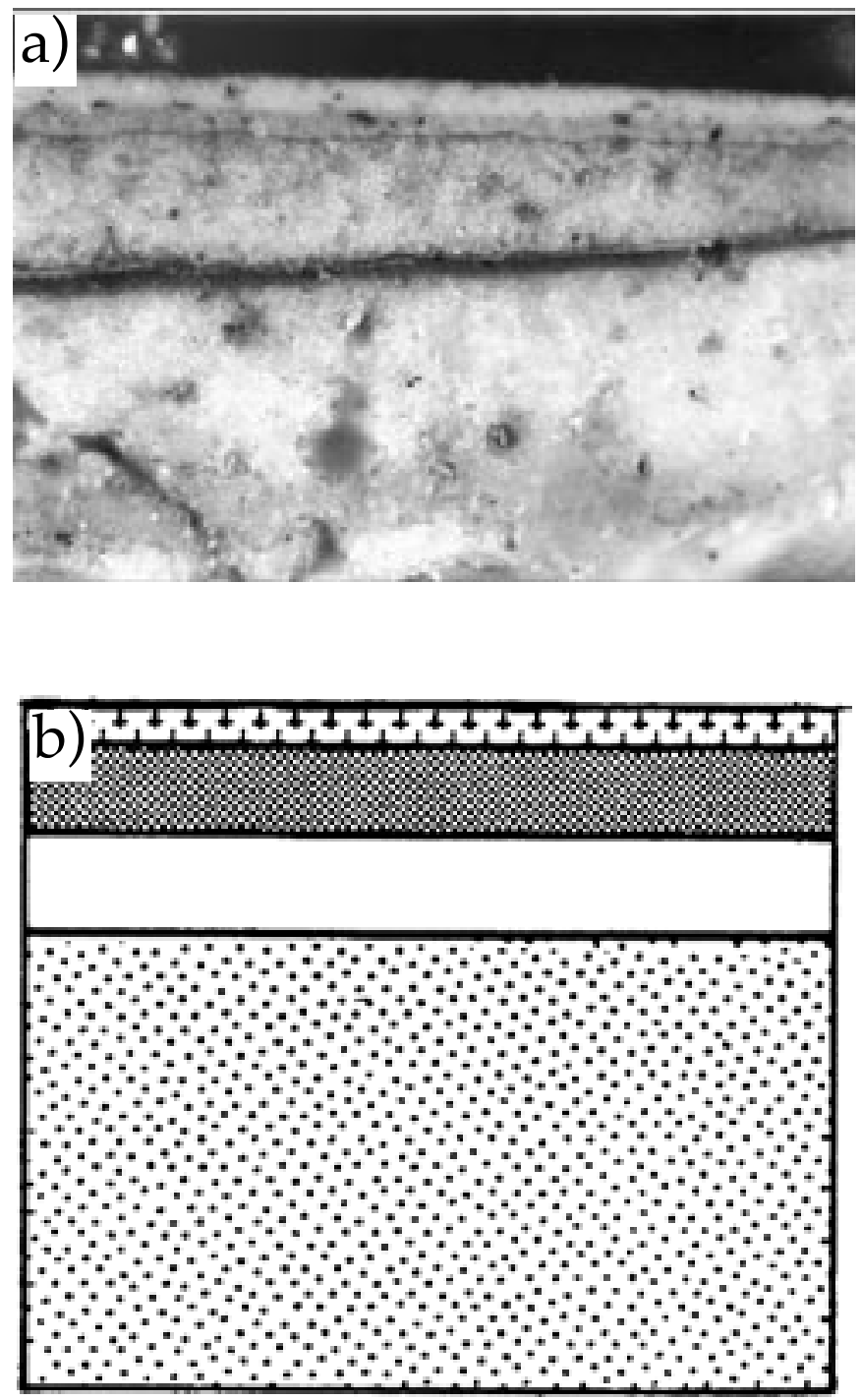

Fig.3.- a) Microfotografía (x70) de una preparación estratigráfica correspondiente a la capa pictórica y soporte de un fragmento de pintura mural; b) Se muestra un esquema general de las distintas capas analizadas en la estratigrafía anterior y sus características más destacadas.
Se pueden encontrar otros ejemplos ilustrativos de secciones estratigráficas en las referencias bibliográficas de este artículo. En lo que respecta a materiales del Patrimonio Cultural investigados según el procedimiento descrito, cabe citar como caso particular, el análisis de una sección estratigráfica de una muestra de pintura mural (11). Mediante estudio por microscopía óptica de algunas estratigrafías, se han observado unos granos blanquecinos. Se trata, probablemente, de mármol triturado y mezclado que es el soporte de la pintura. Es importante mencionar que debajo de esta capa se encuentra otra (conocida como ariccio) donde se aprecian, además de cal, granos de arena que presentan coloración. Asimismo, análisis químicos por EDX han comprobado la presencia del elemento Calcio y los resultados obtenidos mediante espectroscopía IR que se han llevado a cabo en este estudio de pigmentos y soportes, han demostrado la presencia de silicatos, donde también destaca la cantidad de carbonato de calcio que serviría de aglutinante. Se concluye, pues, que el segundo tipo de mortero está realizado con una sola capa, superpuesta a la de pigmentos, encima de las cuáles se volvió a pintar. El mortero está compuesto por cal y granos de arena tal y como se deduce por su composición química según datos de EDX.

Por otra parte, si se encuentran muestras de pintura sobre tabla, donde además de los estratos aparezca el soporte de tabla de madera, a la hora de realizar las estratigrafías se procurará hacer, además de un primer corte vertical a la muestra, otro horizontal a los vasos leñosos del propio soporte de madera. De esta forma, en otra fase de estudio podría incluso determinarse el tipo de madera utilizada.

En definitiva, con los análisis posteriores realizados a partir de las secciones estratigráficas, se puede llegar a conocer la naturaleza química de los pigmentos utilizados en las pinturas murales y los posibles aglutinantes, así como conseguir más información sobre distintos aspectos que caracterizan las muestras originales, como son los soportes en cuanto a tipología, estructura y número de capas de morteros. Por ejemplo, estos resultados revelarían con mayor seguridad la técnica utilizada en pinturas murales elegidas en un determinado muestreo (por ejemplos, técnicas "a fresco" o "a secco"), como ya se ha mencionado, mostrando un ejemplo ilustrativo en la Figura 2, o bien si lo han sido con la técnica al óleo $(6,7,9,10)$ o al temple, como se ha descrito anteriormente y se ha puesto de manifiesto en el ejemplo de la Figura 3.

\section{SUMARIO Y CONSIDERACIONES FINALES}

En apartados anteriores se han expuesto los aspectos prácticos más destacados en el análisis de estratos que se pueden realizar en obras del Patrimonio Cultural; en particular, los pigmentos y soportes como ejemplos más ilustrativos, dentro del campo de la Cerámica y el Vidrio. Al disponer de muestras muy pequeñas, heterogéneas y relativamente frágiles, es necesario realizar una inclusión de las mismas empleando una resina, de preferencia incolora, que además sirve de soporte para una serie de manipulaciones mecánicas que dan lugar a la preparación de la sección estratigráfica.

Por medio de la microscopía óptica se pueden realizar una serie de observaciones directamente sobre los diferentes estratos y la secuencia que se pueden encontrar en una muestra dada, además de obtener una valiosa información sobre técnicas de ejecución $(6,7,9,10)$ e incluso estado de conservación (9-12). Por consiguiente, las secciones estratigráficas son de suma 
utilidad y lo que se ha pretendido en este artículo es dar una serie de consejos y orientaciones útiles que permitan una fácil, sencilla, económica, relativamente rápida y simultánea, además de versátil metodología técnica para la preparación de secciones estratigraficas.

Se ha descrito la metodología de trabajo para la obtención de estratigrafías que vienen siguiendo los autores, desde hace años. En concreto, las distintas etapas de preparación se pueden resumir en: a) preparación inicial, llevando a cabo pequeños taladros cónicos en tiras de (poli)metacrilato, donde se incluyen los fragmentos a estudiar; b) embutido con un polímero incoloro (compuesto de resina y catalizador); c) corte de las preparaciones; d) lijado y pulido, y e) observación al microscopio óptico o lupa y fotografiado. Asimismo, se han expuesto una serie de ejemplos ilustrativos, resultado de experiencias de los autores, sobre estratigrafías de pigmentos y soportes.

Es necesario destacar que la preparación de estratigrafías abre la posibilidad del estudio posterior por distintas técnicas instrumentales de análisis de materiales, obteniendo información de relevancia, por ejemplo sobre textura, composición química y de fases, etc. de muestras tan valiosas.

Por último, hay que indicar que la preparación de secciones estratigráficas se puede extender al estudio de materiales cerámicos y vidriados con relativa facilidad siguiendo las indicaciones aquí expuestas. En este sentido, para las investigaciones en diversas temáticas del Patrimonio Cultural, y también como fase previa a un estudio científico que permita la obtención de una amplia información de la propia obra de arte, no cabe duda que es fundamental la preparación de muestras representativas sin alterar el material de partida (fragmentos y/o micromuestras). Todo ello es muy importante, además, si lo que se pretende es llegar a un diagnóstico o aproximación al estado de alteracióndegradación de la obra con objeto de una fase posterior de restauración y conservación de la misma, a cargo de otros especialistas, como legado para el futuro.

\section{AGRADECIMIENTOS}

Se agradece la financiación recibida al Proyecto de investigación del Plan Nacional de Materiales MAT200202549, mencionando que está cofinanciado en parte con Feder, en el marco del cual se ha realizado este trabajo. Asimismo, se agradece a la Junta de Andalucía a través del III Plan Andaluz de Investigación las facilidades dadas para la realización de estos estudios tecnológicos y a la Red Temática RTPHC del CSIC, constituida en 2001, donde está integrado el grupo de investigación al que pertenecen los autores.

\section{BIBLIOGRAFÍA}

1. J. C. Roldán, "La intervención en el Patrimonio. ¿Para quién conservamos?", Cuadernos de Restauración, 1, 5-9 (1999).

2. R. Mayer, "Materiales y técnicas del arte", Edit. Hermann Blume, 687 pp., Madrid, 1988.

3. M. Matteini y A. Moles, "Ciencia y Restauración. Método de investigación”, Ed. Nerea, Junta de Andalucía, Consejería de Cultura, Instituto Andaluz del Patrimonio Histórico. Bilbao, 2001. Nota: se trata de la traducción española, realizada por M. Martínez de Marañón, de la obra italiana de los mismos autores M. Matteini y A. Moles, "La chimica nel restauro. I materiali dell'arte pittorica", Edit. Nardini, 379 pp. Roma, 1989.

4. B. Gómez, M.A. Respaldiza y Mª. L. Pardo (eds.), “III Congreso Nacional de Arqueometría", Serie Colección Abierta número 50. Secretariado de publicaciones, Universidad de Sevilla. Sevilla, 2001.

5. J. Plesters, "Section transversal and chemical analysis of paint samples", Studies in Conservation 1, 110-157 (1956).

6. J. Plester, L. Lazzarini, "Preliminary observations on the technique and materials of Tintoretto", p. 7-26 en: Conservation and Restauration of Pictorial Art, Ed. Butterwhorts, London, 1976.

7. M.C. Gay, "Application of staining methd to section transversal in the study of the media", p. 78-83 en: Conservation and Restauration of Pictorial Art, Ed. Butterwhorts, London, 1976.

8 M. Matteini y A. Moles, "Técnica de la sección transversal y de las láminas delgadas", p. 27-35, Cap. 2, en ref. 3. Nota: Al final de la obra, en la sección "Imágenes en color", pág. 285, aparece una microfotografía de una sección transversal extraida de un fragmento de Tabla del S. XIV, con repinte, que muestra una finísima lámina de oro.

9. A. Ruiz Conde, $M^{a}$ Arjonilla Alvarez, $M^{a}$. E. Enrique Magariño, J.C. Rivero Cabello, R. Fernández López y P. J. Sánchez Soto, “Investigaciones previas sobre pinturas murales encontradas recientemente en la Iglesia parroquial de Nuestra Señora de las Nieves (La Rinconada, Sevilla)", p. 149-150 en: V Congreso Ibérico de Arqueometría, El Puerto de Santa María (Cádiz), 30 Septiembre, 1-3 Octubre 2003, Libro de Resúmenes de Actas, Imprenta San Rafael, Cádiz, 2003.

10. Ma Arjonilla Alvarez, M. González, J. M. Calderón, J. González-González, E. Jiménez Roca, M. A. Avilés, A. Ruiz Conde y P. J. Sánchez Soto, “Estudio científico de la pintura mural de San Cristóbal en el convento de Santa Paula (Sevilla)", p. 157-8 en: V Congreso Ibérico de Arqueometría, El Puerto de Santa María (Cádiz), 30 Septiembre, 1-3 Octubre 2003, Libro de Resúmenes de Actas, Imprenta San Rafael, Cádiz, 2003.

11. A. Kriznar, J. Höfler, A. Ruiz Conde y P. J. Sánchez Soto, “Caracterización arqueométrica de pigmentos y soportes de pinturas murales góticas (S. XIIIXV)", Bol. Soc. Esp. Ceram. V. (aceptado), 2005.

12. $\mathrm{M}^{a} \mathrm{C}$. Jiménez de Haro, J. L. Pérez Rodríguez y A. Justo, “La técnica del brocado para decorar las cerámicas de las puertas de la Catedral de Sevilla", p. 325-333, en: III Congreso Nacional de Arqueometría, Eds. B. Gómez, M.A. Respaldiza y Mª.L. Pardo, Serie Colección Abierta número 50. Secretariado de publicaciones, Universidad de Sevilla. Sevilla, 2001.
Recibido: 30.07 .04

Aceptado: 30.03 .05 